\title{
Novel targeted therapies: the future of rheumatoid arthritis? Mavrilumab and tabalumab as examples
}

\author{
Bernard Combe, ${ }^{1}$ Ronald van Vollenhoven ${ }^{2}$
}

Rheumatoid arthritis (RA) control and outcome has greatly improved with the efficacy of biologic disease-modifying antirheumatic drugs (DMARDs) ${ }^{1}$ combined with early effective management and treatment targeting remission or low disease activity. ${ }^{23}$

However, although biologic therapyblocking key molecules involved in the pathogenesis of RA has greatly improved RA management, some needs are still unmet. About $30 \%$ of patients with RA show inadequate response to the first biologic therapy, ${ }^{4-8}$ which is usually a tumour necrosis factor (TNF) blocker, currently considered with methotrexate (MTX) as the 'gold standard' in RA therapy. ${ }^{1}$ After failure of at least one TNF inhibitor, the response rate of biological agents is $20-40 \%$ after adjustment for the placebo response. ${ }^{9}$ In addition, the response may decrease over time in some patients. Finally, only a few patients achieve remission or low disease activity, the rate being lower for established than early RA. ${ }^{2}$

Since the remarkable but partial success of TNF inhibitors, tremendous efforts have been made to offer additional treatment options for RA patients. Thus, several biologics drugs with other modes of action have been developed, which block cytokines other than TNF or cells involved in the pathogenesis of RA. ${ }^{10}$ This has led to the licensing of biological DMARDs inhibiting interleukin 1 (IL-1) (anakinra), IL-6 receptor (tocilizumab), B cells (rituximab) and T-cell costimulation (abatacept). ${ }^{1}{ }^{3}$ The availability of this variety of biological drugs has offered additional strategies for rheumatologists

\footnotetext{
'Department of Rheumatology, Lapeyronie Hospital Montpellier I University, Montpellier, France

${ }^{2}$ Unit for Clinical Therapy Research Inflammatory Diseases (ClinTRID), Department of Medicine, The Karolinska Institute, Stockholm, Sweden
}

Correspondence to Dr Bernard Combe, Département de Rhumatologie, Hôpital Lapeyronie, Montpellier 34295, France; b-combe@chu-montpellier.fr in daily practice, but refractory patients remain, and many still have active disease and progressive disability. Another 'fashionable' and more recent strategy has been to try to identify biomarkers that could identify subpopulations of patients with better response to a specific drug. ${ }^{11}$ However, biomarkers, so far, have failed to predict the clinical response to any biological or synthetic DMARD at the individual patient level, and personalised medicine, while undoubtedly of great potential and tremendous research and practical interest, still remains a longterm prospect in RA management. ${ }^{12}$

In this issue of Annals of the Rheumatic Diseases, Burmester et $a l^{13}$ and Genovese et al ${ }^{14}{ }^{15}$ report on phase II clinical trials of two new biological agents with novel mechanisms of action. These new biologic DMARDs target granulocytemacrophage colony stimulating factor (GM-CSF) receptor $\alpha$ and B-cell activating factor (BAFF), molecules that may play a role in the pathogenesis of RA. ${ }^{10}$

Mavrilimumab, a human monoclonal antibody anti-GM-CSF receptor $\alpha$, is a competitive antagonist of GM-CSF signalling. While GM-CSF is known to most of us as a growth factor for leukocytes (and used therapeutically as an alternative to G-CSF for the treatment of neutropenia), it also modulates functions of myeloid cells such as macrophages. ${ }^{16}$ Therefore, blockade of GM-CSF could be expected to result in a decrease of leukocyte activation and attenuation of autoimmune inflammation. In a previous report, Burmester et al ${ }^{17}$ evaluated the safety, efficacy and pharmacokinetic and pharmacodynamic profiles of a single infusion of mavrilimumab in a doubleblind, placebo-controlled, dose-escalating study of patients with RA who concomitantly received MTX. This preliminary study suggested encouraging effects of mavrilimumab for RA. In the current study, Burmester et al ${ }^{13}$ evaluated the 12 -week safety and efficacy of subcutaneous mavrilimumab (10, 30, 50 or $100 \mathrm{mg}$ every other week) versus placebo in patients with RA $(n=239)$ with inadequate response to MTX. All patients received background MTX. Treatment with mavrilimumab met the primary endpoint (reduction of DAS28 c-reactive protein $(\mathrm{CRP}) \geq 1.2$ ) as compared with placebo at week 12 . This positive result was mainly driven by the higher dose (100 mg), with clinical effect observed within the first 2 weeks of treatment. At 12 weeks, clinical improvement as measured by American College of Rheumatology (ACR) response rate, was greater for patients $(n=39)$ who received $100 \mathrm{mg}$ mavrilimumab every other week than placebo. ACR20, 50 and 70 response rates were $69.2,30.8$ and $17.9 \%$ versus $40.0,12.0$ and $4.0 \%$, respectively, for patients receiving placebo (intent to treat (ITT) population with non-responder imputation (NRI)). Low disease activity (defined as DAS28 CRP score $\leq 3.2$ ) did not significantly differ between active treatment (30.8\% with $100 \mathrm{mg}$ mavrilimumab) and placebo (20.0\%). CRP level and erythrocyte sedimentation rate differed significantly for mavrilimumab versus placebo. No particular safety signal was noted and, specifically, anti-GM-CSF treatment did not appear to lead to clinically significant leukopenia.

Tabalumab is a human anti-BAFF monoclonal antibody that neutralises both membrane-bound and soluble BAFF (also known as B Lymphocyte Stimulator (BLyS), as TNF- and APOL-related leukocyte expressed ligand (TALL-1) and as CD257). BAFF is a cytokine of the TNF family required for $B$-cell survival. ${ }^{18}$ Although the precise role of $B$ cells in the pathogenesis of RA remains unclear, the established efficacy of rituximab strongly supports the idea that downregulation of (autoreactive) B cells may benefit patients with RA. Genovese et $a l^{14}$ report the results from a 24-week double-blind, placebo-controlled, dose-ranging trial of 158 patients with active RA receiving MTX. Tabalumab (1-120 mg) or placebo was given subcutaneously every 4 weeks. Tabalumab met the primary endpointdose-response relationship based on a statistical model of the proportion of patients achieving ACR50 at week 24-as compared with placebo. At week 12, observed ACR20 and 50 response rates were significantly higher with only the $120 \mathrm{mg}$ tabalumab dose as compared with placebo $(66.7 \%$ and $33.3 \%$ vs $33.3 \%$ and $11.1 \%$, respectively (ITT with NRI)). At week 24, only the ACR50 response 
rate significantly differed between the $120 \mathrm{mg}$ dose and placebo. The response with any other dose did not differ from that with placebo at any time. The change in CRP levels was similar among treatments. The incidence of infections was greater with tabalumab than placebo, with no dose-related effect. No other safety signal was observed. Genovese et al ${ }^{15}$ also reported efficacy and safety of tabalumab in patients with RA with inadequate response to TNF blockers. Patients $(n=100)$ were randomised to receive, with background MTX, placebo or two intravenous doses of tabalumab (30 or $80 \mathrm{mg}$ ) at weeks 0,3 and 6 . The primary endpoint was not met: the authors found no significant difference in ACR50 response between tabalumab treatment $(12.7 \%)$ and placebo $(2.9 \%)$ at week 16. Likewise, ACR20 response rates did not differ between groups: placebo $17.1 \%$, tabalumab (30 mg) 25.7\%, tabalumab (80 mg) $28.6 \%$. Some secondary endpoints such as DAS28-CRP changes did differ. Based on these phase II trials, a phase III trial programme was initiated, but in February 2013, Eli Lilly, the manufacturer of tabalumab announced that it will discontinue the Phase III RA programme for tabalumab due to lack of efficacy in interim analysis. The decision was not based on safety concerns, and the tabalumab Phase III programme for systemic lupus erythematosus is still ongoing and will continue as planned. ${ }^{19}$ These data are in line with previous trials with two other biologic therapies (belimumab, atacicept) that target BAFF, which both showed disappointing clinical responses in patients with RA. ${ }^{20} 21$

The findings from the three trials reported in this issue, as well as some previously published trials, provided initial support for the hypothesis that these two new biological therapies could offer some clinical efficacy compared with placebo, at least in patients with RA with suboptimal response to MTX. However, the magnitudes of the therapeutic responses in these trials were modest, and their clinical relevance was, therefore, not entirely clear. In the case of tabalumab, the phase III trial led to the conclusion that the efficacy was not sufficient to warrant further development. For mavrilimumab the question is still open, and we do recognise that those trials were only phase II, and that the somewhat weak efficacy results may be related to drug dose or methodological issues, including study design, primary endpoints and sample size calculations.
Regardless, the ACR20 and 50 response rates seem modest as compared with those obtained in the phase II trial of the most recent drug approved for $\mathrm{RA}^{22}$ and are in the lower range of the response rates reported for the therapy of currently marketed biologics when added to MTX. $^{23}{ }^{24}$ In addition, a truly effective therapy for RA would be unlikely to miss endpoints such as acute-phase reactant changes. While for the sake of our patients we hope that anti-GMCSF will prove to be 'successful', the results to date only allow more guarded optimism. One does hope that both these novel biologics might find a role as outstanding treatments for certain well defined patient groups within the entire field of rheumatology.

For those who develop antirheumatic drugs, a key question is what level of response in phase II would be sufficient to proceed to phase III: as it turned out in the case of tabalumab, a more pessimistic reading of the phase II results could have avoided the huge expenses involved in the phase III programme and spared the participating patients the 'doublewhammy' of being treated with an ineffective drug while being exposed to potential risks. Unfortunately, the determination when to proceed from phase II to phase III is enormously difficult to make, and some currently available drugs had modest phase II results. Perhaps the most prudent advice would be to follow through on ambiguous phase II results with an additional phase II trial rather than a full-fledged phase III programme.

In a wider perspective, we may have to reconsider our expectations when viewing the development of therapies with novel mechanisms of action for patients with RA. Because of the variety of therapies available for RA, new targeted compounds without clear benefit, compared with existing drugs are unlikely to improve management of RA. Ideally, these new drugs should provide a better benefit:risk ratio for the whole RA population as compared with existing therapies, such as TNF blockers and MTX, or at least provide a clear advantage in a subset of patients such as those with inadequate response to anti-TNF agents. All biological drugs currently available show similar response rates and same frequency of good outcome regardless of their mechanisms of action, with respect to the RA population where they are evaluated (early RA, inadequate response to MTX or TNF). ${ }^{9} 1223$ In addition, attempts to combine two targeted biologic agents have failed to show additional efficacy, while at least two such combinations have demonstrated significantly increased toxicity. $^{25} 26$ These data do not suggest that focusing on the difference in mode of action will improve the therapeutic success for RA.

One of the major unmet needs in RA is the group of patients for whom anti-TNF agents have failed. We could also add the patients with secondary loss of response and the 'partial responders' who still have some active disease despite improvement with first-line biologics. Such patients may be more likely to have less TNF-dependent disease, and could benefit from subsequent treatment with a targeted therapy of different class. However, this attractive hypothesis has still not been confirmed by clinical data, and the different biologics, including other TNF inhibitors, seem to work similarly in patients without response to TNF blockers. ${ }^{9}$ The network involved in the pathogenesis of RA may be so complex, with many interactions between cells and between cytokines, ${ }^{10}$ that targeting different molecules in this network may have a roughly similar effect on the downstream process leading to joint inflammation and destruction. Patient populations with inadequate response to TNF inhibitors are a major challenge for rheumatologists in clinical practice, and future drug developments should focus on therapies for these refractory patients.

The development of biologics that selectively block additional specific cytokines, such as the BAFF-antagonists and GM-CSF antagonists reviewed here, is of considerable theoretical importance, and may yet turn out to very beneficial for certain subsets of patients. For the large populations of patients with RA and other inflammatory arthritides, these developments are more likely to represent rather modest steps forward. True progress beyond cytokine-directed and celldirected immunomodulators will have to come from better strategies to identify patients earlier, and initiation of the appropriate therapeutic strategies as expeditiously as possible.

Competing interests $\mathrm{BC}$ has received honorarium from Lilly, Merck, Novartis, Pfizer, Roche-Chugai, UCB. RvV has received grants and/or honoraria from AbbVie (Abbott), BMS, GSK, Lilly, MSD, Pfizer, Roche, UCB.

Contributors $\mathrm{BC}$ and RvV wrote and approve the manuscript.

Competing interests None. 
Provenance and peer review Not commissioned; externally peer reviewed.

Accepted 8 April 2013

Published Online First 26 April 2013

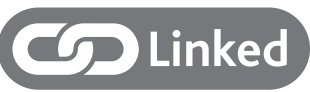

http://dx.doi.org/10.1136/annrheumdis-2012-202450

- http://dx.doi.org/10.1136/annrheumdis-2012-202275

- http://dx.doi.org/10.1136/annrheumdis-2012-202775

- http://dx.doi.org/10.1136/annrheumdis-2012-202864

Ann Rheum Dis 2013;72:1433-1435.

doi:10.1136/annrheumdis-2013-203261

\section{REFERENCES}

1. van Vollenhoven RF. Treatment of rheumatoid arthritis: state of the art 2009. Nat Rev Rheumatol 2009;5:531-41.

2. Smolen JS, Aletaha D, Bijlsma JW, et al. Treating rheumatoid arthritis to target: recommendations of an international task force. Ann Rheum Dis 2010;69:631-7.

3. Smolen JS, Landewé R, Breedveld FC, et al. EULAR recommendations for the management of rheumatoid arthritis with synthetic and biological disease-modifying antirheumatic drugs. Ann Rheum Dis 2010;69:964-75.

4. Weinblatt ME, Keystone EC, Furst DE, et al. Adalimumab, a fully human anti-tumor necrosis factor alpha monoclonal antibody, for the treatment of rheumatoid arthritis in patients taking concomitant methotrexate: the ARMADA trial. Arthritis Rheum 2003:48:35-45.

5. Weinblatt ME, Kremer JM, Bankhurst AD, et al. A trial of etanercept, a recombinant tumor necrosis factor receptor:Fc fusion protein, in patients with rheumatoid arthritis receiving methotrexate. $N$ Engl J Med 1999;340:253-9.

6. Keystone E, Heijde Dv, Mason D Jr, et al. Certolizumab pegol plus methotrexate is significantly more effective than placebo plus methotrexate in active rheumatoid arthritis: findings of a fifty-two-week,phase III, multicenter, randomized, double-blind, placebo-controlled,parallel-group study. Arthritis Rheum 2008;58:3319-29.

7. Kremer JM, Genant HK, Moreland LW, et al. Effects of abatacept in patients with methotrexate-resistant active rheumatoid arthritis: a randomized trial. Ann Intern Med 2006;144:865-76.

8. Smolen JS, Beaulieu A, Rubbert-Roth A, et al. Effect of interleukin-6 receptor inhibition with tocilizumab in patients with rheumatoid arthritis (OPTION study): a double-blind, placebo-controlled, randomised trial. Lancet 2008;371:987-97.

9. Salliot C, Finckh A, Katchamart W, et al. Indirect comparisons of the efficacy of biological antirheumatic agents in rheumatoid arthritis in patients with an inadequate response to conventional disease-modifying antirheumatic drugs or to an anti-tumour necrosis factor agent:a meta-analysis. Ann Rheum Dis 2011;70:266-71.

10. McInnes IB, Schett $G$. The pathogenesis of rheumatoid arthritis. N Engl J Med 2011;365:2205-19.

11. Emery $\mathbf{P}$, Dörner T. Optimising treatment in rheumatoid arthritis: a review of potential biological markers of response. Ann Rheum Dis 2011;70:2063-70.

12. Smolen JS, Aletaha D. Forget personalised medicine and focus on abating disease activity. Ann Rheum Dis 2013;72:3-6.

13. Burmester GR, Weinblatt ME, Mclnnes IB, et al. Efficacy and safety of mavrilimumab in subjects with rheumatoid arthritis. Ann Rheum Dis 2013;72 1445-52.

14. Genovese MC, Lee E, Satterwhite J, et al. A phase 2 dose-ranging study of subcutaneous Tabalumab for the treatment of patients with active rheumatoid arthritis and an inadequate response to methotrexate. Ann Rheum Dis 2013;72 1453-60.

15. Genovese MC, Fleischmann RM, Greenwald M, et al. Tabalumab, an anti-BAFF monoclonal antibody, in patients with active rheumatoid arthritis with an inadequate response to TNF inhibitors. Ann Rheum Dis 2013;72:1461-8.

16. Cornish AL, Campbell IK, McKenzie BS, et al. G-CSF and GM-CSF as therapeutic targets in rheumatoid arthritis. Nat Rev Rheumatol 2009:5:554-9.

17. Burmester GR, Feist E, Sleeman MA, et al. Mavrilimumab, a human monoclonal antibody targeting GM-CSF receptor- $\alpha$, in subjects with rheumatoid arthritis: a randomised, double-blind, placebo-controlled, phase I, first-in-human study. Ann Rheum Dis 2011;70:1542-9.

18. Davidson A. Targeting BAFF in autoimmunity. Curr Opin Immunol 2010;22:732-9.

19. Eli Lilly and Company. Press Release 7 February 2013. http://newsroom.lilly.com/releasedetail.cfm? ReleaselD=738769

20. Genovese M, Filipowicz-Sosnowska A, Merrill J, et al. Differential responsiveness to belimumab in combination with standard of care therapy in RF+ TNF-inhibitor and methotrexate partial responder subgroups of subjects with moderate-severe rheumatoid arthritis. Arthritis Rheum 2005;52: S1989.

21. van Vollenhoven RF, Kinnman $N$, Vincent $E$, et al Atacicept in patients with rheumatoid arthritis and an inadequate response to methotrexate: results of a phase II, randomized, placebo-controlled trial. Arthritis Rheum 2011;63:1782-92.

22. Fleischmann R, Cutolo M, Genovese MC, et al. Phase llb dose-ranging study of the oral JAK inhibitor tofacitinib (CP-690,550) or adalimumab monotherapy versus placebo in patients with active rheumatoid arthritis with an inadequate response to disease-modifying antirheumatic drugs. Arthritis Rheum 2012;64:617-29.

23. Singh JA, Christensen R, Wells GA, et al. A network meta-analysis of randomized controlled trials of biologics for rheumatoid arthritis: a Cochrane overview. CMAJ 2009;181:787-96.

24. Nam JL, Winthrop KL, van Vollenhoven RF, et al. Current evidence for the management of rheumatoid arthritis with biological disease-modifying antirheumatic drugs: a systematic literature review informing the EULAR recommendations for the management of RA. Ann Rheum Dis 2010;69:976-86.

25. Genovese MC, Cohen S, Moreland L, et al. Combination therapy with etanercept and anakinra in the treatment of patients with rheumatoid arthritis who have been treated unsuccessfully with methotrexate. Arthritis Rheum 2004;50:1412-19.

26. Weinblatt M, Schiff M, Goldman A, et al. Selective costimulation modulation using abatacept in patients with active rheumatoid arthritis while receiving etanercept: a randomised clinical trial. Ann Rheum Dis 2007:66:228-34. 